PROCEEDINGS OF THE

AMERICAN MATHEMATICAL SOCIETY

Volume 133, Number 5, Pages 1401-1409

S 0002-9939(04)07656-7

Article electronically published on October 18, 2004

\title{
DUALS AND ENVELOPES OF SOME HARDY-LORENTZ SPACES
}

\author{
MARC LENGFIELD
}

(Communicated by N. Tomczak-Jaegermann)

\begin{abstract}
For $0<p<1$ we describe the dual spaces and Banach envelopes of the spaces $H^{p, q}$ for finite values of $q$ and for $H_{0}^{p, \infty}$, the closure of the polynomials in $H^{p, \infty}$. In addition, we determine the $s$-Banach envelopes for the spaces $H^{p, q}$ in the cases $0<q<p<s \leq 1$ and $0<q<p \leq s \leq 1$.
\end{abstract}

\section{$\S 1$. INTRODUCTION}

For $0<p \leq \infty$, let $H^{p}$ be the usual Hardy space of analytic functions on the unit disc $U$. A well-known theorem of Duren, Romberg, and Shields [4] describes the dual of $H^{p}$ for the range $0<p<1$. This paper presents an extension (Theorem 1 below) of that result to the class $H^{p, q}$ of Hardy-Lorentz spaces on $U$. Determining the dual of a quasinormed linear space $X$ is closely related to determining the Banach envelope, or 1-Banach envelope, of $X$. For $0<p<s<1$ Aleksandrov [1] and Coifman and Rochberg 2 independently determined the $s$-Banach envelope of $H^{p}$. Theorem 2 below does the same for the spaces $H^{p, q}$ if $0<q<p<s \leq 1$ or $0<p<q \leq s \leq 1$. Before stating our results we introduce some definitions and notation.

Let $m$ denote normalized Lebesgue measure on the boundary $\mathbb{T}$ of $U$, and for a measurable function $f$ on $\mathbb{T}$, let $\lambda_{f}$ denote the distribution function of $|f|$ with respect to $m$. That is,

$$
\lambda_{f}(s)=m(\{z \in \mathbb{T}:|f(z)|>s\}), \quad s>0 .
$$

For $0<p, q \leq \infty$ and a function $f$ measurable on $\mathbb{T}$, the Lorentz functional $\|\cdot\|_{p, q}$ is defined at $f$ by

$$
\|f\|_{p, q}=\left(p \int_{0}^{\infty} \lambda_{f}(s)^{q / p} s^{q-1} d s\right)^{1 / q}, \quad q \neq \infty
$$

and

$$
\|f\|_{p, \infty}=\sup _{s>0} s \lambda_{f}(s)^{1 / p}, \quad q=\infty .
$$

Following one of the usual definitions of $H^{p}$, we define the Hardy-Lorentz space $H^{p, q}$ to be the space of functions $f$ in the Smirnov class on $U$ such that the radial limit function $\tilde{f}$ of $f$ satisfies $\|\tilde{f}\|_{p, q}<\infty$. Then $H^{p, q}$ is, in an obvious way, a quasinormed space. We write $H_{0}^{p, \infty}$ for the closure in $H^{p, \infty}$ of the polynomials.

Received by the editors April 1, 2003 and, in revised form, January 7, 2004.

2000 Mathematics Subject Classification. Primary 32A35.

(C)2004 American Mathematical Society Reverts to public domain 28 years from publication 
We begin by defining a class of mixed-norm Bergman spaces: for a continuous function $f$ on $U$ and for $0<r<1$ and $0<p<\infty$ define $M_{p}(r, f)$ to be, as usual,

$$
\left(\frac{1}{2 \pi} \int_{0}^{2 \pi}\left|f\left(r e^{i \theta}\right)\right|^{p} d \theta\right)^{1 / p}
$$

With $M_{\infty}(r, f)$ defined in the standard way, for $0<p, q \leq \infty$ and for $\alpha>0$, let $H(p, q, \alpha)$ be the space of holomorphic functions $f$ on $U$ for which the quasinorm given by

and by

$$
\left(\int_{0}^{1} M_{p}(r, f)^{q}(1-r)^{q \alpha-1} d r\right)^{1 / q} \text { if } q<\infty
$$

$$
\sup \left\{M_{p}(r, f)(1-r)^{\alpha}: 0<r<1\right\} \text { if } q=\infty
$$

is finite. The symbol $H_{0}(p, \infty, \alpha)$ stands for the closure in $H(p, q, \alpha)$ of the polynomials.

For $\beta>0$ and a holomorphic function

$$
f(z)=\sum_{k=0}^{\infty} a_{k} z^{k}
$$

on $U$, recall that the fractional derivative $f^{[\beta]}$ and fractional integral $f_{[\beta]}$ of $f$ are defined by

$$
f^{[\beta]}(z)=\sum_{k=0}^{\infty} \frac{\Gamma(k+\beta+1)}{k !} a_{k} z^{k}, \quad f_{[\beta]}(z)=\sum_{k=0}^{\infty} \frac{k !}{\Gamma(k+\beta+1)} a_{k} z^{k} .
$$

Let $D^{\beta}$ and $D_{\beta}$ be the associated operators so that, e.g., $D^{\beta} f=f^{[\beta]}$.

For $0<p, q \leq \infty$, write $q_{*}=\max \{q, 1\}$ and let $H_{*}^{p, q}=H_{0}^{p, q}$ if $q=\infty, H_{*}^{p, q}=$ $H^{p, q}$ otherwise. In Theorem 1 below the symbol $q_{*}^{\prime}$ denotes the Hölder conjugate of $q_{*}$ defined by the equation

$$
\frac{1}{q_{*}}+\frac{1}{q_{*}^{\prime}}=1
$$

Theorem 1. Fix $0<p<1$ and $0<q \leq \infty$. The dual of $H_{*}^{p, q}$ is $H\left(\infty, q_{*}^{\prime}, 1\right)$ in the following sense:

(i) If $g^{\left[\frac{1}{p}\right]} \in H\left(\infty, q_{*}^{\prime}, 1\right)$ then, with $d \nu(z)$ denoting normalized Lebesgue measure on $U$,

$$
f \mapsto \frac{1}{\Gamma(1 / p)} \int_{U} f(z) g^{\left[\frac{1}{p}\right]}(\bar{z})\left(1-|z|^{2}\right)^{\frac{1}{p}-1} d \nu(z)
$$

defines a bounded linear functional $S_{g}$ on $H_{*}^{p, q}$ and

$$
\left\|S_{g}\right\| \leq C(p, q)\left\|g^{\left[\frac{1}{p}\right]}\right\|_{H\left(\infty, q_{*}^{\prime}, 1\right)} .
$$

(ii) If $\phi$ is a bounded linear functional on $H_{*}^{p, q}$, put $a_{k}=\phi\left(z^{k}\right)$ for $k=0,1, \ldots$ and define

$$
g_{\phi}(z)=\sum_{k=0}^{\infty} a_{k} z^{k}
$$

Then $g_{\phi}^{\left[\frac{1}{p}\right]} \in H\left(\infty, q_{*}^{\prime}, 1\right)$,

$$
\left\|g_{\phi}^{\left[\frac{1}{p}\right]}\right\|_{H\left(\infty, q_{*}^{\prime}, 1\right)} \leq C(p, q)\|\phi\|,
$$


and

$$
\phi(f)=\frac{1}{\Gamma(1 / p)} \int_{U} f(z) g_{\phi}^{\left[\frac{1}{p}\right]}(\bar{z})\left(1-|z|^{2}\right)^{\frac{1}{p}-1} d \nu(z)
$$

for $f \in H_{*}^{p, q}$.

Before stating Theorem 2 we recall the definition of the $p$-Banach envelope of a quasinormed space $X$ : Fix $p \in(0,1)$. For $x \in X$ define

$$
\|x\|_{X_{p}}=\inf \left\{\left(\sum_{n=1}^{N}\left\|x_{n}\right\|_{X}^{p}\right)^{\frac{1}{p}}: x_{n} \in X, x=\sum_{n=1}^{N} x_{n}\right\} .
$$

Then, if $X$ has a separating dual, $\|\cdot\|_{X_{p}}$ is a $p$-norm on $X$, and the completion $[X]_{p}$ of $X$ with respect to $\|\cdot\|_{X_{p}}$ is called the $p$-Banach envelope of $X$. The space $[X]_{p}$ is characterized by the following fact: any continuous linear mapping of $X$ into a complete $p$-normed space $Y$ extends uniquely to a mapping of $[X]_{p}$ into $Y$. If $p=1$, then $[X]_{p}$ is called the Banach envelope of $X$ and, for $x \in X$, the norm $\|x\|_{X_{1}}$ is equivalent to $\sup \left\{|\phi(x)|: \phi \in X^{*},\|\phi\| \leq 1\right\}$. Using Theorem 1 , it can be shown that, for $0<p<1$ and $1<q<\infty$, we have

$$
\left[H^{p, q}\right]_{1}=H(1, q, 1 / p-1) \text { and }\left[H_{0}^{p, \infty}\right]_{1}=H_{0}(1, \infty, 1 / p-1) .
$$

The following result is an analog of this for some other values of the indices. As previously mentioned, the portion of the theorem corresponding to case (ii) is known and due to Aleksandrov [1] and to Coifman and Rochberg [2].

Theorem 2. Fix $p \in(0,1)$ and suppose one of the following holds:

(i) $0<q<p<s \leq 1$,

(ii) $0<p=q<s \leq 1$,

(iii) $0<p<q \leq s \leq 1$.

Then, with equivalent $s$-norms,

$$
\left[H^{p, q}\right]_{s}=H(s, s, 1 / p-1 / s) .
$$

The remainder of this paper is organized as follows: $\S 2$ contains the proofs of Theorems 1 and 2 while $\S 3$ contains proofs of certain lemmas used in $\S 2$.

\section{§2. Proofs of Theorems 1 and 2}

Proof of Theorem 1. We prove (i) first. Suppose $f \in H^{p, q}, g^{\left[\frac{1}{p}\right]} \in H\left(\infty, q_{*}^{\prime}, 1\right)$. Then

$$
\begin{gathered}
\left|S_{g}(f)\right|=\left|\frac{1}{\Gamma(1 / p)} \int_{U} f(z) g^{\left[\frac{1}{p}\right]}(\bar{z})\left(1-|z|^{2}\right)^{\frac{1}{p}-1} d \nu(z)\right| \\
\leq C(p) \int_{0}^{1} M_{1}(r, f)(1-r)^{\left(\frac{1}{p}-1-\frac{1}{q_{*}}\right)} M_{\infty}\left(r, g^{\left[\frac{1}{p}\right]}\right)(1-r)^{\left(1-\frac{1}{q_{*}}\right)} d r \\
\leq C(p)\left(\int_{0}^{1} M_{1}(r, f)^{q_{*}}(1-r)^{q_{*}\left(\frac{1}{p}-1\right)-1} d r\right)^{1 / q_{*}}\left(\int_{0}^{1} M_{\infty}\left(r, g^{\left[\frac{1}{p}\right]}\right)^{q_{*}^{\prime}}(1-r)^{q_{*}^{\prime}-1} d r\right)^{1 / q_{*}^{\prime}} \\
=C(p)\|f\|_{H\left(1, q_{*}, \frac{1}{p}-1\right)}\left\|g^{\left[\frac{1}{p}\right]}\right\|_{H\left(\infty, q_{*}^{\prime}, 1\right)}
\end{gathered}
$$

by Hölder's inequality. Now it follows from Lemma 1 below (proved in $\S 3$ ) that

$$
\|f\|_{H\left(1, q_{*}, \frac{1}{p}-1\right)} \leq C(p, q)\|f\|_{H^{p, q}} .
$$

Thus $S_{g}$ is bounded on $H^{p, q}$ and (1) follows. 
Lemma 1. Suppose $0<p<s \leq \infty, 0<q \leq t \leq \infty$. Then there are the following continuous embeddings:

$$
H^{p, q} \hookrightarrow H(s, t, 1 / p-1 / s)
$$

and

$$
H_{0}^{p, \infty} \hookrightarrow H_{0}(s, \infty, 1 / p-1 / s) .
$$

To prove (ii), let $\phi,\left\{a_{k}\right\}$, and $g_{\phi}$ be as in (ii). A computation shows that (3) holds whenever $f(z)=z^{k}$ for nonnegative integral $k$. Once (2) is established, (3) will follow for all $f \in H_{*}^{p, q}$ from (1) and the density in $H_{*}^{p, q}$ of the polynomials. Thus it remains to prove (2).

The first part of the proof of (2) follows 4. Suppose $f \in H_{*}^{p, q}$ has Taylor series $\sum b_{k} z^{k}$. Suppose, for example, that $\sum b_{k} z^{k}$ converges to $f(z)$ uniformly on $U$. Then it follows that

$$
\phi(f)=\sum_{k=0}^{\infty} a_{k} b_{k} .
$$

In particular, if we take for $f(z)$ the function

$$
c_{\frac{1}{p}, w}(z)=\frac{1}{(1-w z)^{\frac{1}{p}+1}},
$$

it follows that

$$
\left|\frac{g_{\phi}^{\left[\frac{1}{p}\right]}(w)}{\Gamma\left(\frac{1}{p}+1\right)}\right|=\left|\phi\left(c_{\frac{1}{p}, w}\right)\right| \leq\|\phi\|\left\|c_{\frac{1}{p}, w}\right\|_{H^{p, q}} .
$$

A computation shows that

$$
\left\|c_{\frac{1}{p}, w}\right\|_{H^{p, q}} \leq \frac{C(p, q)}{1-|w|} .
$$

Thus

$$
\left|g_{\phi}^{\left[\frac{1}{p}\right]}\right| \leq \frac{C(p, q)\|\phi\|}{1-|w|} .
$$

This gives (2) in the case $q \leq 1$ (and is essentially the argument from [4]). So assume that $1<q \leq \infty$. Since now $q_{*}=q$ it is required to establish

$$
\left\|g_{\phi}^{\left[\frac{1}{p}\right]}\right\|_{H\left(\infty, q^{\prime}, 1\right)} \leq C(p, q)\|\phi\| .
$$

Fix an arbitrary $\epsilon>0$. We begin by estimating

$$
\begin{gathered}
\left\|g_{\phi}^{\left[\frac{1}{p}\right]}\right\|_{H\left(\infty, q^{\prime}, 1\right)}=\left(\sum_{n=1}^{\infty} \int_{1-\epsilon^{n-1}}^{1-\epsilon^{n}} M_{\infty}\left(r, g_{\phi}^{\left[\frac{1}{p}\right]}\right)^{q^{\prime}}(1-r)^{q^{\prime}-1} d r\right)^{\frac{1}{q^{\prime}}} \\
\leq\left(\sum_{n=1}^{\infty} M_{\infty}\left(1-\epsilon^{n}, g_{\phi}^{\left[\frac{1}{p}\right]}\right)^{q^{\prime}} \int_{1-\epsilon^{n-1}}^{1-\epsilon^{n}}(1-r)^{q^{\prime}-1} d r\right)^{\frac{1}{q^{\prime}}} \\
\leq C(\epsilon, q)\left(\sum_{n=1}^{\infty} \epsilon^{n q^{\prime}} M_{\infty}\left(1-\epsilon^{n}, g_{\phi}^{\left[\frac{1}{p}\right]}\right)^{q^{\prime}}\right)^{\frac{1}{q^{\prime}}} .
\end{gathered}
$$

Thus $\left(2^{\prime}\right)$ will follow from

$$
\left(\sum_{n=1}^{\infty} \epsilon^{n q^{\prime}} M_{\infty}\left(1-\epsilon^{n}, g_{\phi}^{\left[\frac{1}{p}\right]}\right)^{q^{\prime}}\right)^{\frac{1}{q^{\prime}}} \leq C(\epsilon, p, q)\|\phi\| .
$$


Now let $\alpha=\left\{\alpha_{n}\right\}$ be an arbitrary sequence in the unit ball of $\ell^{q}$. To establish (4) it is enough to bound

$$
\sum_{n=1}^{\infty}\left|\alpha_{n}\right| \epsilon^{n} M_{\infty}\left(1-\epsilon^{n}, g_{\phi}^{\left[\frac{1}{p}\right]}\right)
$$

by $C(\epsilon, p, q)\|\phi\|$. Choose sequences $\beta=\left\{\beta_{n}\right\}$ and $\xi=\left\{\xi_{n}\right\}$ of unimodular complex numbers such that, if $\sigma_{n}=\alpha_{n} \beta_{n}$ and $w_{n}=\left(1-\epsilon^{n}\right) \xi_{n}$, then (5) has the form

$$
\sum_{n=1}^{\infty} \sigma_{n} \epsilon^{n} g_{\phi}^{\left[\frac{1}{p}\right]}\left(w_{n}\right)
$$

Recall that

$$
c_{\frac{1}{p}, w}(z)=\frac{1}{(1-w z)^{\frac{1}{p}+1}}
$$

and, for any positive integer $N$, define

$$
F_{N}=\Gamma\left(\frac{1}{p}+1\right) \sum_{n=1}^{N} \sigma_{n} \epsilon^{n} c_{\frac{1}{p}, w_{n}} .
$$

We require the following result (which is proved in $\S 3$ ):

Lemma 2. Suppose $0<p \leq 1$. Fix $\epsilon \in(0,1)$ and a sequence $\left\{\xi_{n}\right\}$ of points in the unit circle $\mathbb{T}$. For $n \in \mathbb{N}$ define $w_{n}=\left(1-\epsilon^{n}\right) \xi_{n}$. Then the operator $V$ defined for complex sequences $s=\left\{s_{n}\right\}$ by

$$
V(s)=\sum_{n=1}^{\infty} s_{n} \epsilon^{n} c_{\frac{1}{p}, w_{n}}
$$

satisfies

$$
\|V(s)\|_{H^{p, q}} \leq C(\epsilon, p, q)\|s\|_{\ell^{q}}
$$

if $p \leq q \leq \infty$. If $s \in c_{0}$, then $V(s) \in H_{0}^{p, \infty}$. If $q<\infty$ and $s \in \ell^{q}$ or if $s \in c_{0}$, then the series converges in the appropriate quasinorm.

This result implies that $\left\|F_{N}\right\|_{p, q} \leq C(p, q)$ for all $N$. Thus

$$
\sum_{n=1}^{N} \sigma_{n} \epsilon^{n} g_{\phi}^{\left[\frac{1}{p}\right]}\left(w_{n}\right)=\left|\phi\left(F_{N}\right)\right| \leq\left\|F_{N}\right\|_{p, q}\|\phi\| \leq C(p, q)\|\phi\|
$$

for all $N$. By the choice of $\beta$ and $\xi$, this yields the desired bound for (5) and therefore completes the proof of Theorem 1 .

Proof of Theorem 2. The proof of Theorem 2 requires two lemmas about fractional integration. The first is an extension of a result of Hardy and Littlewood ([6], Theorem 33) and is proved in $\S 3$; the second is a consequence of Theorems 3 and 5 in 9 .

Lemma 3. Suppose $0<p<\infty, 0<q \leq \infty$. For $0<\alpha<1 / p$, let $p(\alpha)$ be defined by

$$
\frac{1}{p(\alpha)}=\frac{1}{p}-\alpha
$$

Then

$$
D_{\alpha}: H^{p, q} \rightarrow H^{p(\alpha), q}
$$


Lemma 4. Suppose $0<p, q \leq \infty, 0<\sigma<\alpha<\infty$, and $\beta>0$. Then the mappings

$$
D^{\beta}: H(p, q, \alpha) \rightarrow H(p, q, \alpha+\beta)
$$

and

$$
D_{\sigma}: H(p, q, \alpha) \rightarrow H(p, q, \alpha-\sigma)
$$

are surjective isomorphisms.

In Theorem 2 consider first the case $0<q<p<s \leq 1$ and put $\alpha=1 / q-1 / p$. By Lemma 2,

$$
D_{\alpha}: H^{q} \rightarrow H^{p, q} .
$$

It is easy to see that this implies the existence of a unique continuous linear extension $\bar{D}_{\alpha}$ of $D_{\alpha}$ satisfying

$$
\bar{D}_{\alpha}:\left[H^{q}\right]_{s} \rightarrow\left[H^{p, q}\right]_{s} .
$$

Also, it is known ([1]) that $\left[H^{q}\right]_{s}=H(s, s, 1 / q-1 / s)$ while it follows from Lemma 4 that $D_{\alpha}$ is an isomorphism of $H(s, s, 1 / q-1 / s)$ onto $H(s, s, 1 / p-1 / s)$. Thus the density of $H^{q}$ in $H(s, s, 1 / q-1 / s)$ implies that

$$
H(s, s, 1 / p-1 / s) \hookrightarrow\left[H^{p, q}\right]_{s} .
$$

Since $0<q<p$, we also have

$$
H^{p, q} \subseteq H^{p}
$$

and so

$$
\left[H^{p, q}\right]_{s} \subseteq\left[H^{p}\right]_{s}=H(s, s, 1 / p-1 / s) .
$$

This completes the proof for the case $0<q<p<s \leq 1$. For the case $0<p<q \leq$ $s \leq 1$, Lemma 1 gives

$$
H^{p} \subseteq H^{p, q} \subseteq H(s, s, 1 / p-1 / s),
$$

and the result follows immediately from $\left[H^{p}\right]_{s}=H(s, s, 1 / p-1 / s)$.

\section{§3. Proofs of SUpporting Results}

The following results about interpolation by the real method will be important tools in this section.

Lemma 5. Suppose $0<p_{j}, q_{j}, q \leq \infty$ for $j=0,1, p_{0} \neq p_{1}$, and $0<\theta<1$. Let

$$
\frac{1}{p}=\frac{1-\theta}{p_{0}}+\frac{\theta}{p_{1}} .
$$

Then, with equivalent quasinorms, we have

$$
\left(H^{p_{0}, q_{0}}, H^{p_{1}, q_{1}}\right)_{\theta, q}=H^{p, q} .
$$

A reference is [7, where the result is proved for spaces of functions on the halfplane and it is noted that the version for the disk follows by conformal invariance. We will also require a similar result for our mixed-norm Bergman spaces.

Lemma 6 ([5], Theorem 2.7 and Corollary 2.8). Suppose $0<p<\infty, 0<q_{j} \leq \infty$ and $0<\alpha_{j}<\infty$ for $j=1,2$ with $\alpha_{0} \neq \alpha_{1}$. Let $0<\theta<1,0<q \leq \infty$, and set

$$
\alpha=(1-\theta) \alpha_{0}+\theta \alpha_{1} .
$$

Then, with equivalent quasinorms, we have

$$
\left(H\left(p, q_{0}, \alpha_{0}\right), H\left(p, q_{1}, \alpha_{1}\right)\right)_{\theta, q}=H(p, q, \alpha) .
$$


Proof of Lemma 1. It is enough to prove (i) in the case $t=q$. If $p=q$, then (i) is a result of Hardy and Littlewood (see [3], Theorem 5.11). In general, choose $p_{1}$ and $p_{2}$ such that $0<p_{1}<p<p_{2}<s$. Interpolating the two embeddings

$$
H^{p_{j}} \hookrightarrow H\left(s, p_{j}, 1 / p_{j}-1 / s\right)
$$

yields

$$
\left(H^{p_{0}}, H^{p_{1}}\right)_{\theta, q} \hookrightarrow\left(H\left(s, p_{0}, 1 / p_{0}-1 / s\right), H\left(s, p_{1}, 1 / p_{1}-1 / s\right)\right)_{\theta, q},
$$

and so Lemmas 5 and 6 give (i). Then (ii) follows also since the polynomials are dense in both $H_{0}^{p, \infty}$ and $H_{0}(s, \infty, 1 / p-1 / s)$.

Proof of Lemma 2. We begin with a lemma for the case $q=\infty$.

Lemma 7. Suppose $0<p \leq 1$ and $0<\epsilon<1$. Fix a sequence $\xi=\left\{\xi_{n}\right\}$ of points in the unit circle $\mathbb{T}$, and let $s=\left\{s_{n}\right\}$ be a sequence in $\ell^{\infty}$. Define $w_{n}=\left(1-\epsilon^{n}\right) \xi_{n}$,

$$
g_{n}(z)=\left|c_{\frac{1}{p}, w_{n}}(z)\right|, G_{1, N}(z)=\sum_{n=1}^{N}\left|s_{n}\right| \epsilon^{n} g_{n}(z), \quad \text { and } G(z)=\sum_{n=1}^{\infty}\left|s_{n}\right| \epsilon^{n} g_{n}(z) \text {. }
$$

Then, recalling that $m$ is normalized Lebesgue measure on $\mathbb{T}$,

$$
G \in L^{p, \infty}(m) \text { and }\|G\|_{p, \infty} \leq C(p)\|s\|_{\ell \infty} .
$$

If $s \in c_{0}$, then

$$
G \in L_{0}^{p, \infty}(m) \text { and } \lim _{N \rightarrow \infty}\left\|G-G_{1, N}\right\|_{p, \infty}=0 .
$$

Proof of Lemma \%. Put

$$
q=\frac{p}{1+p}
$$

The following estimates are not difficult to obtain:

$$
\left\|g_{n}\right\|_{\infty} \leq \epsilon^{-\frac{n}{q}}
$$

and

$$
\left\|g_{n}\right\|_{q, \infty} \leq C
$$

where $C$ will denote a positive constant depending only on $p$ and $\epsilon$ which, however, may increase from line to line. Now, from (6),

$$
\left\|G_{1, N}\right\|_{\infty} \leq \sum_{n=1}^{N}\left|s_{n}\right| \epsilon^{n\left(1-\frac{1}{q}\right)} \leq\|s\|_{\ell^{\infty}} \sum_{n=1}^{N} \epsilon^{-\frac{n}{p}} \leq \frac{C_{1}\|s\|_{\ell \infty}}{\epsilon^{\frac{N}{p}}},
$$

where $C_{1}=1 /\left(1-\epsilon^{1 / p}\right)$. Put

$$
t_{N}=\frac{C_{1}\|s\|_{\ell \infty}}{\epsilon^{\frac{N}{p}}}
$$

and

$$
G_{N, \infty}=G-G_{1, N}
$$

We will estimate the distribution functions $\lambda_{G}$ of $G$ and $\lambda_{G_{N, \infty}}$ of $G_{N, \infty}$. It follows from (8) that

$$
\lambda_{G}(t) \leq \lambda_{G_{N}, \infty}\left(t-t_{N}\right) \text { if } t \geq t_{N}
$$

and so

$$
\lambda_{G}\left(2 t_{N}\right) \leq \lambda_{G_{N, \infty}}\left(t_{N}\right) .
$$


It is proved in [8] that the quasinorm $\|\cdot\|_{q, \infty}$ is $q$-convex. Thus, using (7), there is $C$ such that

$$
\left\|G_{N, \infty}\right\|_{q, \infty}^{q} \leq C \sum_{n=N+1}^{\infty}\left(\left|s_{n}\right|^{q} \epsilon^{n q}\left\|g_{n}\right\|_{q, \infty}^{q}\right) \leq C\left(D_{N}\right)^{q} \sum_{n=N+1}^{\infty} \epsilon^{n q},
$$

where $D_{N}=\sup _{n \geq N}\left|s_{n}\right|$. That is,

$$
\left\|G_{N, \infty}\right\|_{q, \infty}^{q} \leq\left(C D_{N} \epsilon^{N}\right)^{q}
$$

or, equivalently,

$$
t^{q} \lambda_{G_{N, \infty}}(t) \leq\left(C D_{N} \epsilon^{N}\right)^{q}
$$

Thus

$$
\lambda_{G_{N, \infty}}\left(t_{N}\right) \leq\left(\frac{C D_{N} \epsilon^{N}}{t_{N}}\right)^{q}
$$

and so it follows from

$$
\epsilon^{N}=\left(\frac{C_{1}\|s\|_{\ell \infty}}{t_{N}}\right)^{p}
$$

and $q+p q=p$ that

$$
\lambda_{G_{N, \infty}}\left(t_{N}\right) \leq\left(\frac{C D_{N}}{t_{N}}\right)^{q}\left(\frac{C_{1}\|s\|_{\ell_{\infty}}}{t_{N}}\right)^{p q}=\frac{C\left(D_{N}\right)^{q}\|s\|_{\ell_{\infty}}^{p q}}{t_{N}^{p}} .
$$

Because

$$
t_{N+1}=\frac{t_{N}}{\epsilon^{\frac{1}{p}}}
$$

we see, using (9), that

(10)

$$
\sup _{2 t_{N} \leq t \leq 2 t_{N+1}} t^{p} \lambda_{G}(t) \leq\left(2 t_{N+1}\right)^{p} \lambda_{G_{N, \infty}}\left(t_{N}\right)=\frac{2^{p}}{\epsilon}\left(t_{N}\right)^{p} \lambda_{G_{N, \infty}}\left(t_{N}\right) \leq C\left(D_{N}\right)^{q}\|s\|_{\ell^{\infty}}^{p q} .
$$

Since

and also

$$
\left(D_{N}\right)^{q}\|s\|_{\ell^{\infty}}^{p q} \leq\|s\|_{\ell^{\infty}}^{p}
$$

$$
\sup _{0 \leq t \leq 2 t_{1}} t^{p} \lambda_{G}(t) \leq\left(2 t_{1}\right)^{p}=C\|s\|_{\ell \infty}^{p}
$$

it follows that

$$
\|G\|_{p, \infty}^{p}=\sup _{t \geq 0} t^{p} \lambda_{G}(t) \leq C\|s\|_{\ell^{\infty}}^{p} .
$$

This is (i) of Lemma 7. If $s \in c_{0}$, then $G \in L_{0}^{p, \infty}(m)$ follows from (10), while

$$
\lim _{N \rightarrow \infty}\left\|G-G_{1, N}\right\|_{p, \infty}=0
$$

follows by applying (i) to $G_{N, \infty}$. This completes the proof of Lemma 7 .

Returning to the proof of Lemma 2 while maintaining the notation of Lemma 7, it is not difficult to check that

$$
\left\|g_{n}\right\|_{p} \leq C \epsilon^{-n} .
$$

The $p$-subadditivity of $\|\cdot\|_{p}$ therefore gives

$$
\|G\|_{p} \leq C\|s\|_{\ell^{p}} .
$$


Interpolating this with the result of Lemma 7 yields

$$
\|G\|_{p, q} \leq C(\epsilon, p, q)\|s\|_{\ell q},
$$

which gives Lemma 2.

Proof of Lemma 3. The proof of Lemma 3 parallels the proof of Lemma 1: The corresponding version for $H^{p}$ spaces is due to Hardy and Littlewood [6], and the present result follows from interpolation and Lemma 5.

\section{REFERENCES}

1. A.B. Aleksandrov, Essays on nonlocally convex Hardy classes, Complex analysis and spectral theory, ed. V.P. Havin and N.K. Nikolskii, Springer Lecture Notes in Math. 864 (1981), 1-89. MR0643380 (84h:46066)

2. R.R. Coifman and R. Rochberg, Representation theorems for holomorphic and harmonic functions in $L^{p}$, Astérique 77 (1980), 110-150. MR0604369 (82j:32015)

3. P.L. Duren, Theory of $H^{p}$ spaces, Academic Press (1970). MR0268655 (42:3552)

4. P.L. Duren, B.W. Romberg, A. Shields, Linear functionals on $H^{p}$ spaces with $0<p<1$, J. Reine Angew. Math. 238 (1969), 32-60. MR0259579 (41:4217)

5. J. Fabrega and J.M. Ortega, Mixed norm spaces and interpolation, Studia Math. 109 (1994), 233-254. MR 1274011 (95j:46021)

6. G.H. Hardy and J.E. Littlewood, Some properties of fractional integrals, II, Math. Z. 34 (1932), 403-439.

7. P.W. Jones, $L^{\infty}$ estimates for the $\bar{\partial}$ problem in the half-plane, Acta Math. 150 (1983), 137152. MR0697611 (84g:35135)

8. N.J. Kalton, Linear operators on $L^{p}$ spaces for $0<p<1$, Trans. Amer. Math. Soc. 259 (1980), 319-355. MR0567084 (81d:47022)

9. J.H. Shi, On the rate of growth of the means $M_{p}$ of holomorphic and pluriharmonic functions on bounded symmetric domains of $\mathbb{C}^{n}$, J. Math. Anal. Appl. 26 (1987), 161-175. MR0900536 (89d:32011)

Department of Mathematics, Florida State University, Tallahassee, Florida 323064510

E-mail address: mlang@math.fsu.edu

Current address: Department of Mathematics, Western Kentucky University, Bowling Green, Kentucky 42101

E-mail address: marc.lengfield@wku.edu 